\title{
A New Way to Understand and Address Women's Health in the Context of Complex Systems: Self-organizing Criticality
}

\author{
Peggy Mancuso ${ }^{1}$, Fuqin Liu ${ }^{1 *}$, Elizabeth Restrepo ${ }^{1}$, Patricia Hamilton ${ }^{1}$, Paolo Grigolini ${ }^{2}$, Lin Zou ${ }^{3}$
}

$\mathrm{I}$ n 2009, the American College of Obstetricians and Gynecologists recommended beginning cervical cancer screening at age 21 (1). After this policy went into effect, there was a significant decrease in screening for chlamydia in females aged 15 to 21 years (2). This unintended decrease in chlamydia screening occurred despite recommendations promoting chlamydia screening. Why should a policy that was intended to promote screening for one disease initiate decreased compliance with screening for another? Women's health professionals could better understand riddles such as this one by applying concepts from nonlinear science developed and employed by physicists, economists, and meteorologists (3).

The complexity of health and health care has been demonstrated (4). Until now, however, our conceptual models and research tools have failed to capture this complexity adequately. As researchers, we too often focus our attention on individual variables, and then we introduce change while holding "background" conditions constant. Our focus is limited to predict specific outcomes caused by our single change while "background conditions" are key to understanding complex systems. We tend to ignore the fact that there is an interacting effect occurring between individual variables and the system in which the variables exist. We may resign ourselves to our errors in predicting the effects of treatments or programs by blaming the constraints of our observations. We tell ourselves that if we took more precise measurements of more variables, we could predict with greater accuracy. The flaws of this mode of thinking were formally described by the meteorologist, Edward Lorenz (5), in the following way:

Two states differing by imperceptible amounts may eventually evolve into two considerably different states...If, then, there is any error whatever in observing the present state-and in any real system such errors seem inevitable - an acceptable prediction of an instantaneous state in the distant future may well be impossible.... In view of the inevitable inaccuracy and incompleteness of weather observations, precise very-long-range forecasting would seem to be nonexistent (p. 133).

Lorenz's insights came to be called the "Butterfly Effect."
In popular terms, a butterfly flapping its wings in Brazil could conceivably start a chain of atmospheric interactions that would result in a tornado in Texas. According to Lorenz, our limitations in predicting future events are Fuqin Liu, PhD, RN, is
an assistant professor
at Texas Woman's
University College of
Nursing in Denton.
Her overall program of
research is related to
maternal and child health
promotion, specifically
preconception health practices and policies
that shape global women's health care. Her
current research is looking at reproductive
health issues among abused women. not solely that we have insufficient data or computing power. Rather, constantly changing, interlocking, systems on many scales (e.g. from insects to jet streams or, in the case of health care, from bacteria to corporations) produce outcomes influenced by the tiniest alteration in the processes involved. Neither unlimited supercomputing power nor massive data sets describing the tiniest detail of every feature of every system involved would enable us to predict exactly what the effect of a single action or intervention would be or where it could be observed. Nevertheless, all is not lost.

Our research team now includes not only health professionals but also experts in economics and physics. This strategy allows us to investigate the coupling of dynamic systems and their emergent properties. Self-organizing criticality is a term introduced by Per Bak in 1996 in his book, How Nature Works. Self-organizing criticality refers to the view that the world around us is not simply as made up of building blocks, but rather formed by interacting processes. It is the processes themselves that hold the key to the changing (often unpredictable) forces that create health and influence systems of care for women (3).

The important subject of complexity in health and health care systems can be dealt with from within this theoretical perspective by making the reasonable assumption that health care involves multiple interacting network layers, economical, psychological and so on. This brings us to the emerging field of multilayer networks that is attracting an exponentially increasing number of researchers all over the world. All these concepts are illustrated in the paper by Grigolini and colleagues (6).

Colleagues both inside and outside of health care are need- 
ed in order to discover ways to recognize and work with the interacting processes at work in any type of change in the health and health care of women. Take nurse staffing, for example. At the simplest level of understanding, we know that adding one more patient to a medical-surgical nurse's usual six-patient work load increases risk of patient mortality (7). However, applying the notion of self-organizing criticality, we acknowledge that the imposition of strict ratios of patients to nurse will start a cascade of both intended and unintended consequences much as the new policy on cervical cancer screening resulted in less screening for chlamydia.

To demonstrate these effects, we will assume a hospital chooses to meet the target ratios by hiring more nurses. Unfortunately, the only nurses available are new graduates and nurses educated in other countries. Relying upon new graduates introduces new challenges and processes. For example, Parker and her colleagues (8) studied newly graduated nurses in Australian hospitals where one interviewee explained:

We were expected to perform and provide care like other nurses that had been there for 20 years! Same amount of patients, and sometimes the most difficult ones because no-one wanted them, and then you would get in trouble for not having completed all the necessary tasks by the end of the day shift (p. 153).

Neff and colleagues (9) found that employing nurses educated outside the United States increased rates of post-surgical 30-day mortality except where patient-to-nurse ratios were lower than average. They concluded that "...U.S. hospitals should give priority to achieving adequate nurse staffing levels, and be wary of hiring large percentages of non-US-educated nurses unless patient-to-nurse ratios are low" (p.366).

In another scenario, the local nursing workforce might be severely limited due to budget constraints on nursing education programs. Hospital administrators may feel their only option for reducing the risks from inadequate nurse staffing is to delegate more care to unlicensed care workers, creating yet another set of problems. Roch et al. (10) conducted a mixed-methods study in Canada and found that when workloads were already heavy, “...collaboration between team members was sometimes reduced to nurses' monitoring patients through nursing assistants without actually supervising or coordinating the monitoring, to the detriment of nurses' performance of caring practices" (p. 235).

Alternatively, hospitals might choose to attract more experienced or better-educated nurses by increasing nurses' salaries, driving up labor costs to meet budget targets. In response, they reduce the number of support staff. In this case, the net result would be that nurses still are unable to attend adequately to their patients' needs because they are taking on the tasks formerly carried out by support personnel.

Clearly, adequately addressing the nurse staffing issue requires recognizing that workforce dynamics, economic incentives, sociological factors such as immigration and migration, health policy, and professional education are not just "background" but integral forces that will interact to create either positive or negative outcomes from our actions. These scenarios also demonstrate that examining not only intended but also unintended consequences is essential. This approach will be of great importance in translational research as it relates to women's health.

The journal, Annals of Family Medicine, has instituted a "learning community" for sharing human insight into the process of turning research evidence into practice. The Journal is engaging diverse participants “...in thinking critically about important issues affecting primary care and then acting on those discussions." They call their approach RADICAL (Read, Ask, Discuss, Inquire, Collaborate, Act, and Learn). The Journal hosts a virtual journal club that accompanies each issue. In the September/October 2015 issue they seek discussion around "Implementation Science is Challenging: When RCT Evidence Doesn't Translate into Practice." The editor explains,

Dissemination/implementation of evidence-based interventions into practice has spawned its own field of work. According to the authors of this study, the collaborative care model for depression is supported by at least 79 randomized controlled trials. So why didn't it work as well as expected when scaled up by these experienced, highly successful implementers? This (Annals Journal Club) provides a chance to answer that question and to develop broader understanding of the challenges of improving patient outcomes in real world practice settings.

The "real world" mentioned here is the world Bak (3) described. The journal is employing social media to explore the self-organizing criticality of health and health care (albeit without calling it that). Readers examine published research and, cognizant of its limitations, they apply their expertise and personal experiences of living within a complex world to imagine the "severe weather" that could develop when the research is implemented in specific settings. That "severe weather" may be unintended effects of women's health practice guidelines or choice of strategies for improving nurse staffing. This radical approach is not in opposition to present-day research as it relates to women's health and health care, rather it is a vital extension of its usefulness. As a group of researchers who aim to further the work in the area of birth outcomes in the complex system, we embrace this new way of thinking.

\section{Ethical issues}

Not applicable.

\section{Conflict of interests}

The authors have no conflicts of interest to disclose.

\section{References}

1. American College of Obstetricians and Gynecologists Committee on Practice Bulletins-Gynecology. ACOG practice bulletin no. 109: cervical cytology screen. Obstet Gynecol. 2009;114(6):1409-1420. 
2. Ursu A, Sen A, Ruffin M. Impact of cervical cancer screening guidelines on screening for chlamydia. Ann Fam Med. 2015;13(4):361-363.

3. Bak P. How nature works: the science of self-organized criticality. New York: Copernicus Press; 1996.

4. Griffiths DF. Complexity science and its relevance for primary health care research. Ann Fam Med. 2007;5(4):377-378. doi:10.1370/afm.727.

5. Lorenz EN. Deterministic nonperiodic flow. J Atmos Sci. 1969;20:130-141.

6. Grigolini P, Piccinini N, Svenkeson A, Pramukkul P, Lambert D, West BJ. From neural and social cooperation to the global emergence of cognition. Front Bioeng Biotechnol. 2015;3(78):1-15. doi:10.3389/fbioe.2015.00078.

7. Aiken LH, Clarke SP, Sloane DM, Sochalski J, Silber
JH. Hospital nurse staffing and patient mortality, nurse burnout, and job dissatisfaction. JAMA. 2002;288(16):1987-1993.

8. Parker V, Giles M, Lantry G, McMillian M. New graduate nurses' experiences in their first year of practice. Nurse Educ Today. 2014;34(1):150-156. doi: 10.1016/j.nedt.2012.07.003.

9. Neff DF, Cimiotti J, Sloane DM, Aiken LH. Utilization of non-US educated nurses in US hospitals: Implications for hospital mortality. Int J Qual Health Care. 2013;25(4):366-372. doi:10.1093/intqhc/ mzt042.

10. Roch G, Dubois CA, Clarke SP. Organizational climate and hospital nurses' caring practices: a mixedmethods study. Res Nurs Health. 2014;37(3):229-240. doi:10.1002/nur.21596.

Copyright $\odot 2016$ The Author(s); This is an open-access article distributed under the terms of the Creative Commons Attribution License (http://creativecommons.org/licenses/by/4.0), which permits unrestricted use, distribution, and reproduction in any medium, provided the original work is properly cited. 